\title{
Omnidirectional inductive wireless charging of a 3D receiver cube inside a box
}

\author{
Sergkei Kamotesov ${ }^{1,2}$, Philippe Lombard ${ }^{2}$, Vincent Semet ${ }^{2}$, Bruno Allard ${ }^{2}$, Maël Moguedet ${ }^{1}$ and Michel Cabrera ${ }^{2}$ \\ ${ }^{1}$ Smart Plastic Products (S2P), 5 Rue Pierre et Marie CURIE, Belignat - BP 21107, 01111 Oyonnax Cedex, France \\ ${ }^{2}$ Université de Lyon, Université Claude Bernard Lyon 1, CNRS, INSA Lyon, Ecole Centrale de Lyon, Ampère, \\ F-69621, Villeurbanne, France \\ Email: sergkei.kamotesov@s-2p.com; mael.moguedet@s-2p.com; michel.cabrera@insa-lyon.fr
}

\begin{abstract}
Omnidirectional inductive wireless charging of a $6 \times 6 \times 6 \mathrm{~cm}^{3} 3 \mathrm{D}$ receiver cube with 6 planar coils inside a $47 \times 47 \times 47 \mathrm{~cm}^{3}$ box with 4 planar emitting coils is studied. The power delivered to the cube in the central position of the box is in the $1 \mathrm{~W}-2.4 \mathrm{~W}$ range with $6-7 \%$ transfer efficiency. In this position, the power is insensitive to the pitch angular orientation. When moving the 3D receiver cube toward the corners of the box, there is no sudden drop of transmitted power. However, the delivered power near the corners increases or decreases in an acceptable range depending on the orientations of the magnetic field of the emitters. Perspectives of this work, when replacing the manufacturing of coils based on PCBs technology by 3D Molded Interconnect Device technology, are briefly discussed.

Index Terms - wireless power transmission (WPT), omnidirectional, magnetic induction, mobile receiver, molded interconnect device
\end{abstract}

\section{INTRODUCTION}

Easy-to-use solutions are needed for powering the increasing number of electronic devices. Among the available Wireless Power Transmission (WPT) technologies, magnetic induction is the most promising one because of its simplicity and power capability [1]. This technology has already spread on the consumer market [2] driven by standards like Qi, PMA and Rezence. So far, these standards offer unidirectional solutions to power stationary devices. Moreover, it is well known that the receiver should be in the vicinity of the emitter and that the energy transfer efficiency is very sensitive to angular misalignment.

In addition, there are many applications where many electronic devices have to be charged while freely moving [3]-[4]. Therefore, in this work we want to analyze a situation where the receiver can move along different directions.

So far, in the literature, the reports deal with the powering of a movable device with an unidirectional array of coils or the powering of a stationary object by emitting uniformly in different directions [5]-[7] but none has combined both. In many cases, the receiver is confronted to limited freedom of movement or to sudden changes of the transmitted power.

That is why in this paper we want to analyze a solution for powering multiple targets in movement along different directions. This is possible by emitting inductive power in many directions with a $3 \mathrm{D}$ array of coils and by receiving it with a 3D array of coils.

\section{EXPERIMENTAL SET UP}

\section{A. 3D Receiver Cube and Inductive Power Box}

As shown in Fig. 1, the experimental set up is composed of a $47 \times 47 \times 47 \mathrm{~cm}^{3}$ box with 4 planar emitting coils on the sides named TRX1 to TRX4 (2-turns with $30 \mathrm{~cm}$ diameter) with a Qfactor of 80 at $6.78 \mathrm{MHz}$. A copper Litz wire of $2 \mathrm{~mm}^{2}$ crosssection was used in order to minimize skin effects.

On the receiving side, there is a $6 \times 6 \times 6 \mathrm{~cm}^{3} 3 \mathrm{D}$ cube with 6 planar coils with a Q-factor of 170 at $6.78 \mathrm{MHz}$ (8-turns with $5 \mathrm{~cm}$ diameter of copper tracks of $70 \mu \mathrm{m}$ thickness, $800 \mu \mathrm{m}$ width and $400 \mu \mathrm{m}$ spacing) made on two-dimensional Printed Circuit Boards (PCB) and named RX1 to RX6.

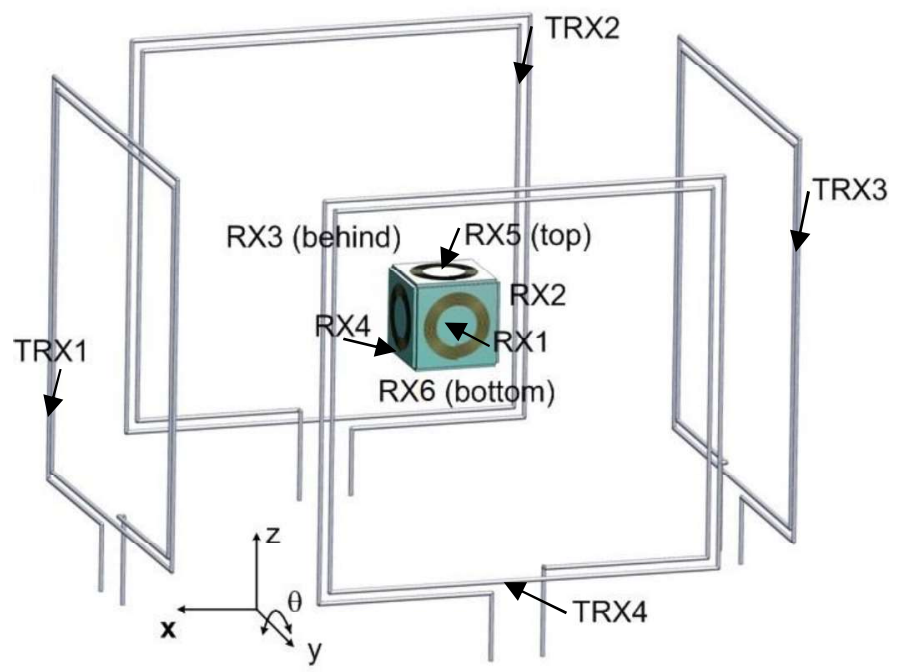

Fig. 1. Schematic diagram of the 3D receiver cube inside the inductive box at central position. In this particular position :TRX1//RX4; TRX4//RX1; TRX3//RX2; TRX2//RX3 (note the presence of top RX5 and bottom RX6). During the experiments, the $3 \mathrm{D}$ receiver will be translated inside the inductive box and/or turned with $\theta$ pitch rotation (see Fig. 3).

The receiver cube is obtained by assembling the 6 planar coils as shown in Fig. 2 (the coils are on the inner faces of the cube).

Ferrite sheets with a surface of $6 \times 6 \mathrm{~cm}^{2}$ and $0.4 \mathrm{~mm}$ thickness are also placed on all inner faces of the cube directly on the coils 
in order to decrease the dependence between the receiving sides. The imaginary and real parts relative permeability of this ferrite are respectively 160 and below 5 for a frequency of $6.78 \mathrm{MHz}$.

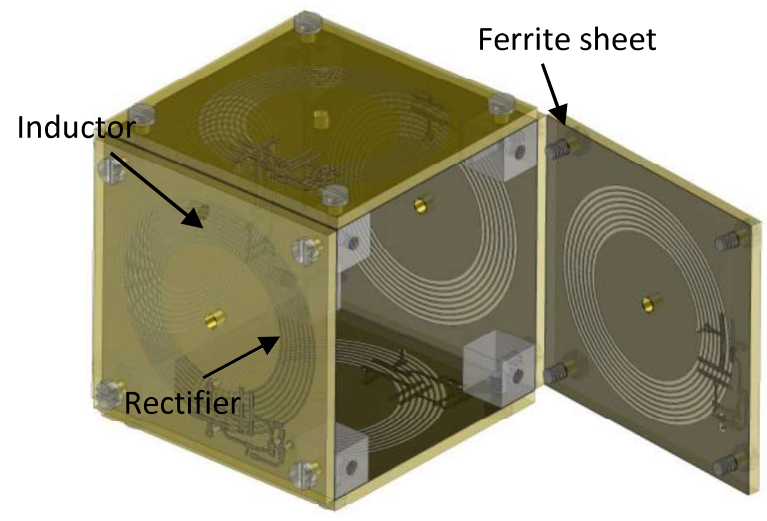

Fig. 2. Transparent $\mathrm{CAD}$ view of the $3 \mathrm{D}$ receiver cube with the planar coils and the electronic rectifiers (see Fig. 4) on PCBs before final assembling. One side is opened to see the inside of the box.

A XYZ translation stage with $\theta$ pitch rotation made of only polymer materials allows to manually move the cube inside the box. For reducing unwanted dissipation, there is no metal in the experimental set up, except some wires for measurement. There is no power storage device in the receiver cube so that the received power is directly used by the load and measured.

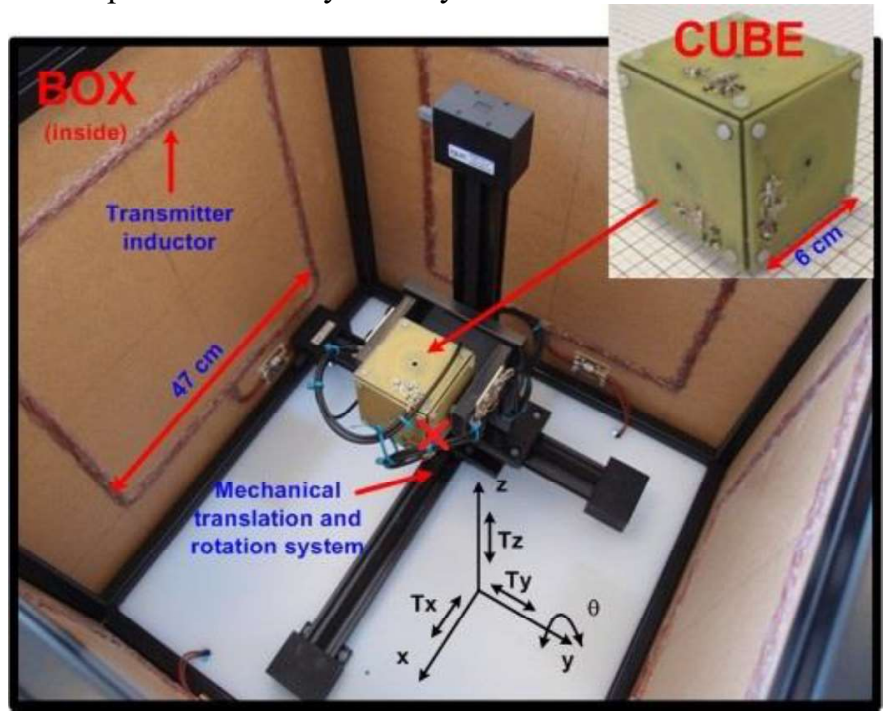

Fig. 3. Experimental set up with the XYZ $\theta$ polymer stage allowing moving the $3 \mathrm{D}$ cube inside the emitter box (no emitter coils on the top and bottom sides of the box). The red cross indicates the origin $(\mathrm{x}=0, \mathrm{y}=0, \mathrm{z}=0)$ that will be used for measurements.

Each transmitter has a $4.42 \mu \mathrm{H}$ inductor $L_{t x}$ in series with a high voltage tuning capacitor $C_{t x}$ as shown on Fig. 4. Each of these LC emitting circuits are powered by a class-D ZVS amplifier working at $6.78 \mathrm{MHz}$ with GAN technology.
On the receiving side, each coil $L_{r x}$ is in parallel with a tuning capacitor $C p_{r x}$ and in series with a capacitor $C s_{r x}$. The voltage is then rectified with Schottky diodes and directly applied to a load $R_{L}$. The value $R_{L}$ of $68 \Omega$ has been used to maximize output power for most of the receivers positions in the box as checked experimentally. Of course, it might be not optimal for some positions. Future improvements will require adapting the charge to the position.

In this system, all the inductors are coupled to each other and share a mutual inductance. Due to the small distance between the transmitting inductors $L_{t \times 1}$ to $L_{t x 4}$, they share a high mutual inductance $M_{t i j}$ and have a dependent resonant frequency. Same thing for $L_{r x 1}$ to $L_{r x 6}$. However, all the receiving and transmitting coils have a very low mutual inductance $M_{\text {trik }}$ because of the difference in size, which makes them almost independent from each other. This means that, once the system has been tuned, the receiver can move everywhere in the box without disturbing the resonance of the emitter.

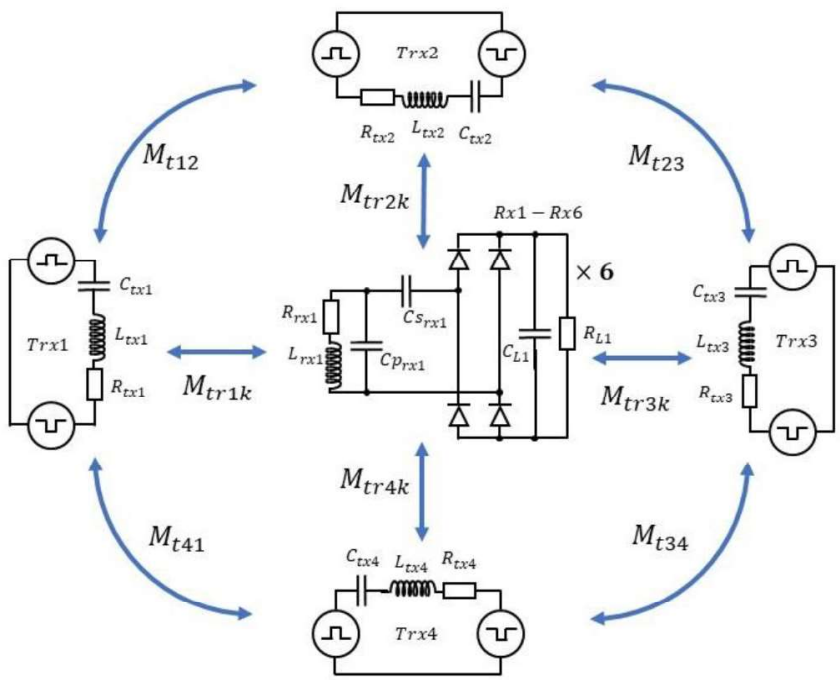

Fig. 4 Equivalent electronic circuit of the transmitter and the receiver. Note that the 6 receivers RX1 to RX6 share a mutual inductance $M r_{i j}$ which is not represented on the schematic.

\section{EXPERIMENTAL RESULTS}

\section{A. Available power transmitted at central position}

Each amplifier supplies approximately $4 \mathrm{~W}$ of power (depending on the position of the receiver) to its transmitter. Transmitting and receiving inductors are tuned to resonance with variable capacitors. The direction of the magnetic field at a given moment is shown on Fig. 5. This configuration is expected to offer a good distribution of power in the box. However, positions of high and low energy (hot and cold spots) are also expected as shown in Fig. 5. At central place $(\mathrm{X}=\mathrm{Y}=\mathrm{Z}=0$ and $\theta=0^{\circ}$ ), the measured Power Delivered to Load (PDL) is $1.03 \mathrm{~W}$ and the Power Transfer Efficiency (PTE) is $6.8 \%$ for $15.2 \mathrm{~W}$ transmitted in total. 


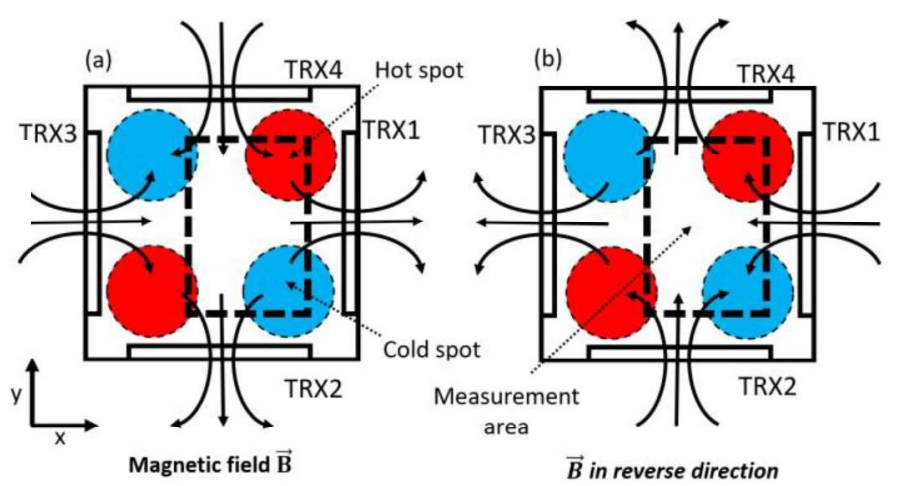

Fig. 5. (a) Directions of magnetic field during the first semi-period of sinwave excitation showing the positions of high and low energy (hot and cold spots). (b) Directions of magnetic field during the second semi-period of sinwave excitation showing that the positions of hot and cold spots remain unchanged.

\section{B. XY cartography at 3 different $z$ values}

The size of the box is $47 \mathrm{x} 47 \mathrm{x} 47 \mathrm{~cm}^{3}$ with origin in the center, which is sufficient for allowing the movement of the $6 \times 6 \times 6 \mathrm{~cm}^{3}$ target along different directions. We do limit the translational movement of the target in the $30 \times 30 \times 30 \mathrm{~cm}$ region, which correspond to the size of the emitting coils. However, due to experimental limitations (in the assembly of polymer translation stages), the $-150 \mathrm{~mm} \leq \mathrm{x} \leq-25 \mathrm{~mm}$ is not accessible for measurement.

So, the objective of the experiment is to measure the power wirelessly transferred to the receiver while moving the cube inside the box with translational movements in the following range:

$$
\begin{gathered}
-25 \mathrm{~mm} \leq \mathrm{x} \leq 150 \mathrm{~mm} \\
-150 \mathrm{~mm} \leq \mathrm{y} \leq 150 \mathrm{~mm}
\end{gathered}
$$

PDL and PTE measurement at $3 \mathrm{z}$-heights are shown in Fig. 6, Fig. 7 and Fig. $8\left(\theta=0^{\circ}\right)$. The area of measurement in the xy directions is shown as a dashed box in Fig. 5 .
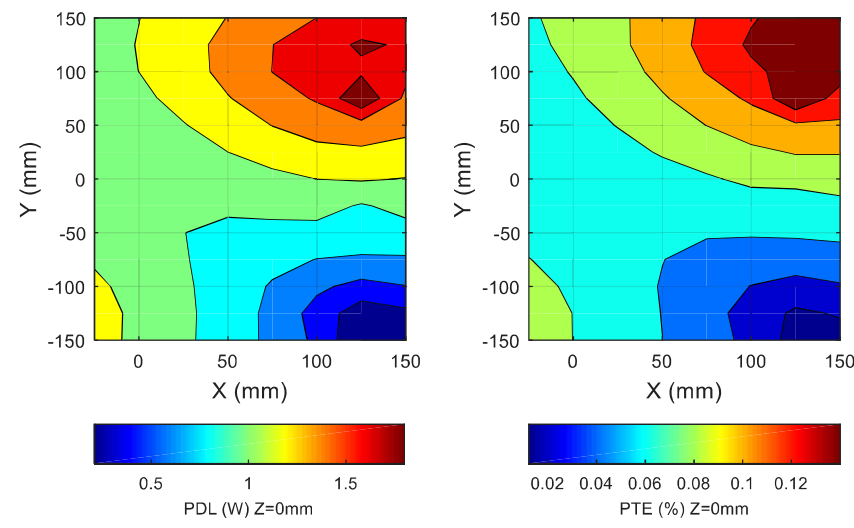

Fig. 6. Power PDL delivered to load and power transfer efficiency PTE at $\mathrm{z}=0 \mathrm{~mm}$.
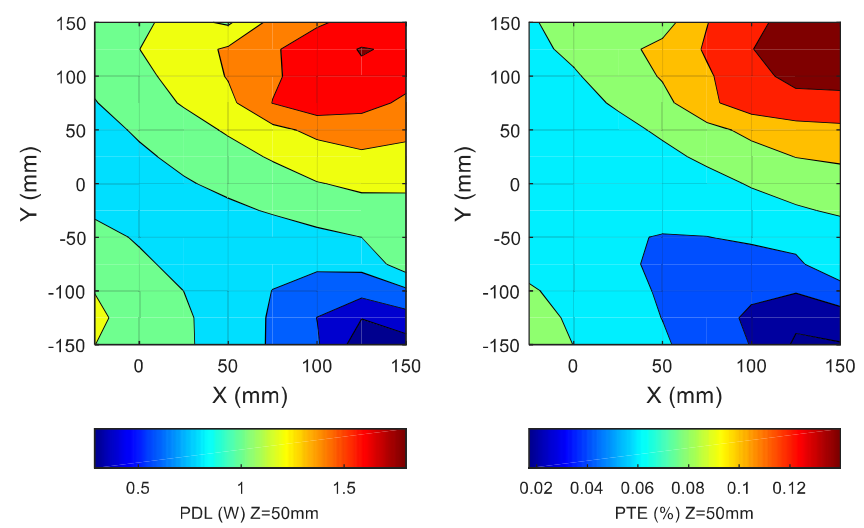

Fig. 7. Power PDL delivered to load and power transfer efficiency PTE at $\mathrm{z}=50 \mathrm{~mm}$
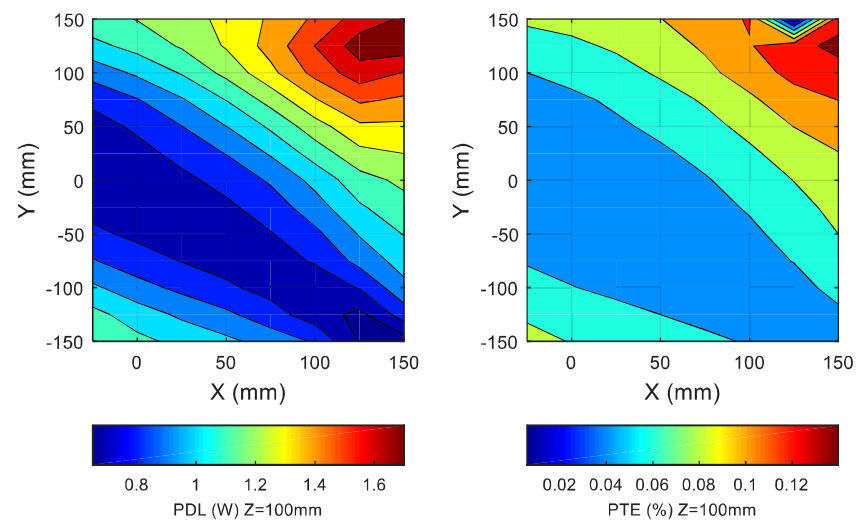

Fig. 8. Power PDL delivered to load and power transfer efficiency PTE at $\mathrm{z}=100 \mathrm{~mm}$.

Measurement results shows that the receiver has almost $1 \mathrm{~W}$ PDL on most of the positions. As expected there are 2 cold points in the corners where the power drops near $0.21 \mathrm{~W}$ and 2 hot points where the power increase up to $1.75 \mathrm{~W}$.

\section{Available power at central position for different pitch}

For the cube at central position, PDL and PTE are measured at different angular pitch $\theta$ within the $-90^{\circ}$ to $90^{\circ}$.range. The values of PDL are relatively constant with $1.04 \mathrm{~W}$ mean value and $30 \mathrm{~mW}$ mean standard deviation. The mean value and mean standard deviation for PTE are respectively $6.68 \%$ and $0.15 \%$. So our conclusion, is that thanks to the design, the transmitted power at central position is almost independent of the orientation.

\section{Available power at central position at increasing input power}

Fig. 10 shows the variation of PDL and PTE at increasing input power. The maximum PDL that we obtained at the central position is $2.43 \mathrm{~W}$ with PTE $6.2 \%$. The actual limit comes from 
the heat dissipation of the amplifier, which has not been yet optimized.
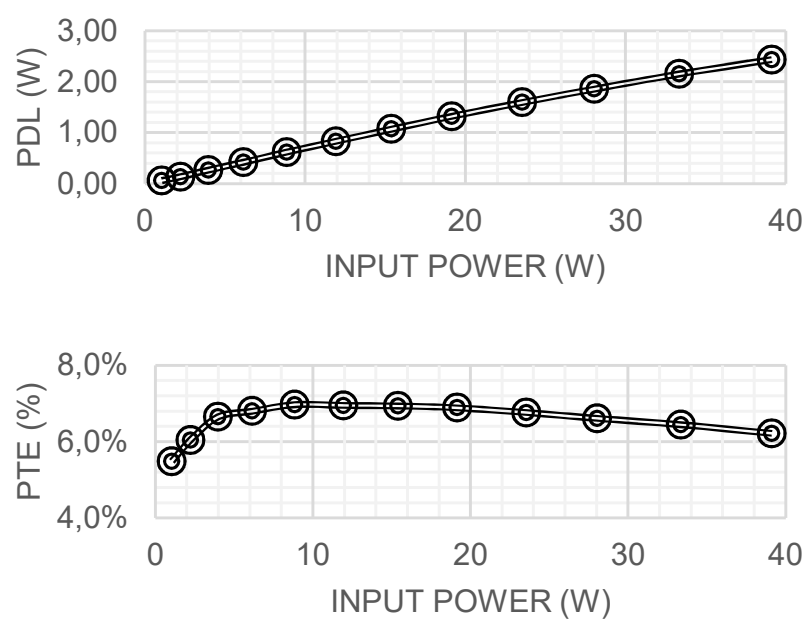

Fig. 10. Measured PDL and PTE at the central position ( $\mathrm{x}=\mathrm{y}=\mathrm{z}=0$ and $\theta=0^{\circ}$ ) for different input power

\section{E. Powering multiple receivers inside the box}

Fig. 11 shows qualitatively the capability of the system to power multiple moving devices. Two planar coils with 4 LEDs tuned at $6.78 \mathrm{MHz}$ were moved inside the box. As discussed below in the conclusion, the next step will be to make use of the Molded Interconnect Device technology (3D MID) to power the LEDs with coils of different orientation.

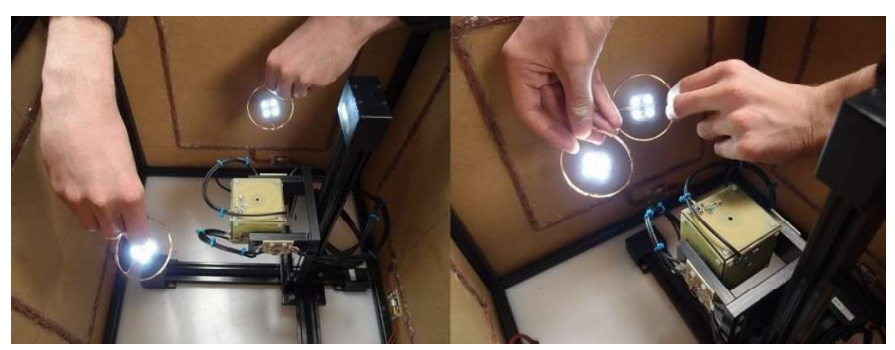

Fig. 11. Two planar coils with 4 LEDs moved inside the box.

\section{CONCLUSION}

Our conclusion is that the power delivered to the $3 \mathrm{D}$ receiver cube in the center is in the $1 \mathrm{~W}-2.4 \mathrm{~W}$ range with $6-7 \%$ transfer efficiency. In this position, the power is insensitive to the orientation. This level of power is interesting for practical applications, for example medical applications.

When moving the $3 \mathrm{D}$ receiver cube toward the corners of the box, the delivered power increases or decreases depending on the orientation of the magnetic field of the emitters. This can probably be improved with a better design. However, there is no sudden drop of transmitted power.
With the experimental set up, powering of multiple moving devices with changes of positional and angular orientation has been qualitatively demonstrated.

The next step will be to use a technology more convenient than conventional PCBs to fabricate the coils of the receiver [8]. Multidirectional receivers require coils with a 3D design that can be made with the 3D MID technology [9]. Previous work showed a way for designing high quality 3D MID inductors [10]. In addition, 3D MID, which allows (relatively) easy integration of 3D inductance coils at the surface of everyday objects. This could be a valuable solution at industrial scale.

\section{ACKNOWLEDGMENT}

The Agence Nationale de la Recherche Technologique (ANRT) is acknowldeged for S. Kamotesov's CIFRE PhD scholarship.

\section{REFERENCES}

[1] Agbinya, Johnson I. Wireless power transfer. Vol. 45. River Publishers, 2015.

[2] Lu, Xiao, Dusit Niyato, Ping Wang, Dong In Kim, and Zhu Han. "Wireless charger networking for mobile devices: Fundamentals, standards, and applications." IEEE Wireless Communications 22, no. 2 (2015): 126-135.

[3] Xu, Qi, Zhaolong Gao, Hao Wang, Jiping He, Zhi-Hong Mao, and Mingui Sun. "Batteries not included: A mat-based wireless power transfer system for implantable medical devices as a moving target." IEEE Microwave Magazine 14, no. 2 (2013): 63-72.

[4] Karpelson, Michael, Benjamin H. Waters, Benjamin Goldberg, Brody Mahoney, Onur Ozcan, Andrew Baisch, Pierre-Marie Meyitang, Joshua R. Smith, and Robert J. Wood. "A wirelessly powered, biologically inspired ambulatory microrobot." In Robotics and Automation (ICRA), 2014 IEEE International Conference on, pp. 2384-2391. IEEE, 2014.

[5] Agbinya, Johnson I., and Nagi F. Ali Mohamed. "Design and study of multi-dimensional wireless power transfer transmission systems and architectures." International Journal of Electrical Power \& Energy Systems 63 (2014): 1047-1056.

[6] Ha-Van, Nam, and Chulhun Seo. "A novel cubic transmitter for multidirectional wireless power transfer." In Wireless Power Transfer Conference (WPTC), 2015 IEEE, pp. 1-3. IEEE, 2015.

[7] Kuo, Ron-Chi, Patrick Riehl, and Jenshan Lin. "3-D wireless charging system with flexible receiver coil alignment." In Wireless Power Transfer Conference (WPTC), 2016 IEEE, pp. 1-4. IEEE, 2016.

[8] Sergkei Kamotesov, Philippe Lombard, Vincent Semet, Bruno Allard, Maël Moguedet, Michel Cabrera. "The Potential of 3D MID Technology for Omnidirectional Inductive Wireless Power Transfer." In Molded Interconnect Devices (MID), 2018 13th International Congress. "Submitted"

[9] Frank, Jorg. "Three-Dimensional Molded Interconnect Devices (3DMID)." Hanser publications (2014).

[10] Sergkei Kamotesov, Philippe Lombard, Christian Vollaire, Vincent Semet, Michel Cabrera, Rabah Dahmani, Amaury Veille, and Maël Moguedet. "Modelization and characterization of 2D and 3D mid inductors for multidirectional inductive proximity sensing." In Molded Interconnect Devices (MID), 2016 12th International Congress, pp. 1-6. IEEE, 2016. 